Bio - grafia. Escritos sobre la Biología y su Enseñanza. ISSN 2027

Edición Extraordinaria. p.p. 503 - 512

Memorias del VIII Encuentro Nacional de Experiencias en Enseñanza de la Biología y la Educación Ambiental. III Congreso Nacional de Investigación en Enseñanza de la Biología.

\title{
UNA ESTRATEGIA DE EDUCACION AMBIENTAL EN LA ESCUELA DENOMINADA "ZOOM EN MI ENTORNO"
}

Oscar Hernando García Cadena ${ }^{1}$ Jenny Johanna Duarte Diaz ${ }^{2}$

\section{RESUMEN}

La estrategia de educación ambiental en la escuela titulada "Zoom en mi entorno", surge como una alternativa para retomar el ámbito ambiental desde una perspectiva diferente a la tradicional, buscando que los estudiantes construyan producciones audiovisuales y diseñen herramientas visuales que generen en la comunidad educativa conciencia frente al manejo de los recursos de su entorno inmediato. Particularmente se buscó establecer sentido de pertenencia y apropiación del ambiente, desde la idea que todos podemos ser superhéroes ambientales en la sociedad en la que vivimos.

N

La estrategia se desarrolló en una línea, que se venía abordando en la institución años atrás llamada "el ambiente desde la comunicación" en la que se propuso el diseño de herramientas audiovisuales ligadas a la visión ambiental de la comunidad (Duarte, et al., 2013). Sin embargo a medida que la propuesta se ha desarrollado se ha centrado en la construcción de recursos audiovisuales y visuales en busca de propiciar una educación ambiental diferente a la tradicional, que permita el cuidado y reconocimiento del entorno.

De esta manera, se ha planteado la construcción de una propuesta de innovación cuya idea se ha centrado en el diseño e implementación de estrategias que propicien la educación ambiental, desde el reconocimiento de sus problemáticas ambientales, la identificación de su entorno y la utilización de los medios de comunicación y las redes sociales para el cuidado del ambiente, cuyo objetivo gira en la transformación de la manera de llevar el mensaje del cuidado del ambiente, realizándolo por medios audiovisuales y visuales llamativos para los miembros de la comunidad.

1 Licenciado en Educación Física. Estudiante de Maestría de Ciencias y Tecnologías del deporte y actividad Física. Universidad Manuela Beltrán. Docente Educación Física y Deportes. Secretaria de educación Distrital. CED La Concepción. oscargarciacadena@hotmail.com

2 Licenciada en Biología. Estudiante del Doctorado Interinstitucional de Educación, Universidad Pedagógica Nacional. Secretaria de educación Distrital. CED La Concepción. jeky1983@gmail.com 
Bio - grafia. Escritos sobre la Biología y su Enseñanza. ISSN 2027

Edición Extraordinaria. p.p. 503 - 512

Memorias del VIII Encuentro Nacional de Experiencias en Enseñanza de la Biología y la Educación Ambiental. III Congreso Nacional de Investigación en Enseñanza de la Biología.

\section{PALABRAS CLAVE}

Educación ambiental, Herramientas visuales y audiovisuales, conciencia ambiental.

\section{ABSTRACT \\ KEYWORDS}

Environmental education, visual and audiovisual tools, environmental awareness.

\section{INTRODUCCION}

Durante la experiencia escolar planteada, se ha identificado que la construcción de estrategias visuales y audiovisuales que promuevan el cuidado del ambiente, por parte de los propios agentes de la comunidad educativa han generado una mayor incidencia en cuanto al cambio de actitudes, en los diversos miembros, en relación a aquellos ejercicios de Educación Ambiental que tradicionalmente se venían adelantando desde el aula, los cuales particularmente estaban limitados a una disciplina de las Ciencias Naturales. De esta forma se ha podido reconocer que la educación ambiental en la escuela debe establecerse desde propuestas diferentes a las tradicionales que partan de la construcción de diversos campos del saber y no centrarse en la realización de actividades desligadas de un propósito de enseñanza.

Así, apoyados en la idea de Gadotti (2000) en la que expone que vivimos en una era de la información próspera, de realidad virtual, de Internet, de sistemas de producción automatizados y de entretenimiento. Reconocemos que las nuevas tecnologías de información y de comunicación han marcado nuestro siglo, transformando los modos de vida, además compartimos la posición en la que se plantea que el desarrollo de la información, ya sea respecto a las fuentes, o en la capacidad de difusión ha generado una verdadera revolución que afecta principalmente la educación y la formación (Gadotti, 2000).

En concordancia con lo anterior y con el objeto de llevar un mensaje de cuidado del ambiente y protección de los recursos que se encuentran en el entorno inmediato de la 
Bio - grafia. Escritos sobre la Biología y su Enseñanza. ISSN 2027

Edición Extraordinaria. p.p. 503 - 512

Memorias del VIII Encuentro Nacional de Experiencias en Enseñanza de la Biología y la Educación Ambiental. III Congreso Nacional de Investigación en Enseñanza de la Biología.

comunidad educativa del Colegio la Concepción, se han desarrollado estrategias comunicativas que garantizan el fortalecimiento de la educación ambiental, de forma alternativa a la tradicional en los diferentes miembros de la comunidad. Lo que puede resultar aplicable a cualquier tipo de población ya que en los diferentes contextos se identifican problemáticas ambientales similares en las que la escuela puede pensar en realizar una intervención que varíe las formas tradicionales de trabajo en Educación Ambiental.

Durante todo el desarrollo, la propuesta investigativa se ha vinculado a las políticas distritales en el marco del Proyecto de Educación para la Ciudadanía y la Convivencia (PECC), el cual propone "promover acciones pedagógicas que permitan proponer proyectos educativos que incidan, no solo en el nivel institucional, sino también en la transformación de las realidades ambientales de su entorno" (SED, 2013). Además, se ha tenido en cuenta el Proyecto Educativo Institucional que propone "ofrecer una formación integral que favorezca el desarrollo de una gestión en que se den las condiciones necesarias para el ejercicio progresivo de competencias que potencien la formación de ciudadanos líderes capaces de razonar, debatir, producir y responder con acierto a la problemática personal, familiar, ambiental y social de nuestra Comunidad" (Documento PEI, 2009).

\section{METODOLOGIA}

Con un enfoque interpretativo se genera una propuesta escolar que retoma como antecedente un trabajo investigativo adelantado previamente en el Centro Educativo Distrital La Concepción en el que se caracterizaron las representaciones sociales de los miembros de la comunidad educativa del concepto ambiente y educación ambiental, determinando que las visiones se encontraban relacionadas en una mayor proporción a las imágenes construidas desde los diferentes medios de comunicación (Duarte y Vallejo 2013). Desde esta conclusión y apoyados en la idea de Barbero (2002) en la que plantea que "el lugar de la cultura en la sociedad cambia cuando la mediación tecnológica de la comunicación deja de ser meramente instrumental para espesarse, densificarse y convertirse en estructural. Pues, la tecnología remite hoy no a la novedad de unos aparatos sino a nuevos modos de percepción y de lenguaje, a nuevas sensibilidades y escrituras". Nos propusimos diseñar herramientas tecnológicas audiovisuales y visuales que permitieran variar las formas de trabajo en educación ambiental. 
Bio - grafia. Escritos sobre la Biología y su Enseñanza. ISSN 2027

Edición Extraordinaria. p.p. 503 - 512

Memorias del VIII Encuentro Nacional de Experiencias en Enseñanza de la Biología y la Educación Ambiental. III Congreso Nacional de Investigación en Enseñanza de la Biología.

En el desarrollo de esta propuesta se ha beneficiado a la comunidad educativa del colegio La Concepción principalmente los estudiantes pertenecientes al proyecto ambiental, que se han integrado de forma voluntaria, estos han sido multiplicadores de la información con sus compañeros, los cuales a su vez han replicado con los miembros de su familia, vecinos y las personas que habitan cerca de la institución. Es muy importante resaltar que dado que las intervenciones realizadas por el grupo permiten el beneficio de este sector, los padres de familia y los docentes han sido permeados por los procesos de transformación evidenciando cambios significativos en el cuidado de los recursos. Adicional, se debe mencionar que el trabajo se ha adelantado en espacios totalmente diferentes a las aulas cotidianas en donde los tiempos de reuniones y actividades con estudiantes se han acordado en jornada extra clase, esto a fin de no centrar la educación ambiental a contenidos propios de algunos conocimientos disciplinares.

\section{Fases de la propuesta}

En el desarrollo de la propuesta se plantearon diversas etapas, algunas de las cuales se han dado secuencialmente y otras de forma simultánea. La primera etapa se describió en la idea de entender que tradicionalmente la comunidad educativa estaba permeada por la educación ambiental que se abordaba de diversas formas, sin embargo se identificó que esta podría ser más asertiva en sus fines, cuando se implicaban medios de comunicación para su enseñanza y de esta manera se replanteo la manera en la que se trabajaría el mensaje ambiental en la escuela.

Así, se propuso con los estudiantes empezar a utilizar lenguajes diferentes y elaborar distintas herramientas comunicativas desde la producción, visual y audiovisual, consiguiendo la construcción de afiches, calcomanías, botones y manillas ambientales referentes a los objetivos del proyecto en desarrollo. Vinculado a esta idea como producto sobresaliente, durante la evolución de la propuesta surgió la idea de crear un súper héroe ambiental que tenía como objetivo inicial presentarse a la comunidad educativa e invitar a los estudiantes a ser parte del grupo ambiental institucional. Por su acogida en los estudiantes, se propuso la construcción de un video clip en el que el súper héroe llevara el mensaje de cuidado del ambiente, no obstante durante la ejecución de esta idea surgió la posibilidad de postulación a una convocatoria realizada por el Canal Capital para la producción de mini series escolares, a la cual se accedió e inicio un proceso de formación continua, con la 
Bio - grafia. Escritos sobre la Biología y su Enseñanza. ISSN 2027

Edición Extraordinaria. p.p. 503 - 512

Memorias del VIII Encuentro Nacional de Experiencias en Enseñanza de la Biología y la Educación Ambiental. III Congreso Nacional de Investigación en Enseñanza de la Biología.

participación de estudiantes integrantes del proyecto ambiental institucional, en esta etapa se construyó un guion literario y técnico de la historia ambiental a abordar. Las capacitaciones fueron realizadas por el equipo de producción del Canal Capital y este proceso fue limitado exclusivamente a la formación de estudiantes en el manejo de herramientas de producción audiovisual.

Posteriormente, se llevaron a cabo las filmaciones de la miniserie durante varias semanas con la participación de los estudiantes y docentes como actores, las cuales fueron dirigidas por el grupo de producción del Canal Capital y enmarcadas en la construcción del guion previamente realizada por los estudiantes. Finalmente, se realizó la post producción, edición del material y publicación del producto en televisión y en páginas de internet como YouTube. De esta forma surgió el diseño de una producción audiovisual propia del colegio La Concepción, la cual se concretó con la producción de la mini serie llamada "El capitán ambiente".

Es importante mencionar que durante el proceso de producción de la miniserie se recurrió a la realización de dibujos tipo historieta por parte de los estudiantes, para reemplazar escenas importantes que no podían ser filmadas por la gran cantidad de efectos especiales que contenían. Y bajo esta idea, se originó la última etapa en el desarrollo de la estrategia, explicada en la propuesta de realizar una cartilla ambiental para colorear dirigida particularmente a estudiantes de primaria, cuyo diseño estuvo a cargo de estudiantes miembros del comité ambiental. En su esquema general se conservaron aspectos relevantes de la producción audiovisual, no obstante se profundizo en problemáticas ambientales actuales a nivel mundial como son: la contaminación del agua, el maltrato animal, el manejo inadecuado de residuos, la tala indiscriminada de árboles con fines económicos y la contaminación del aire.

Esta nueva herramienta se socializo buscando que los niños se concientizaran de las problemáticas ambientales y generaran el cuidado de los recursos, utilizando como lema "Despertar el súper héroe ambiental que cada uno tiene dentro".

\section{RESULTADOS}

En relación a los resultados obtenidos con el desarrollo de la estrategia de educación ambiental debemos iniciar mencionado que en primer lugar obtuvimos la identificación de la 
Bio - grafia. Escritos sobre la Biología y su Enseñanza. ISSN 2027

Edición Extraordinaria. p.p. 503 - 512

Memorias del VIII Encuentro Nacional de Experiencias en Enseñanza de la Biología y la Educación Ambiental. III Congreso Nacional de Investigación en Enseñanza de la Biología.

forma en la que la institución educativa La concepción había venido abordando la educación ambiental, a fin de establecer y delimitar la estrategia. Para esto, nos apoyamos en el planteamiento de González (2000) quien expone que han existido diferentes momentos por lo que ha pasado la incorporación de la dimensión ambiental en el contexto escolar. El autor presenta como primera etapa la llamada, resistencia de la asociación de la educación ambiental a la escuela, considerando que ya hace parte del currículo desde las ciencias naturales; la segunda etapa se explica en la incursión de la educación ambiental como una asignatura particular con un enfoque muy cercano a las ciencias naturales; la tercera etapa se refiere a la ambientalización de las ciencias naturales proponiendo añadir contenidos relacionados con el ambiente en las distintas áreas; y la última etapa se presenta en las propuestas transversales en las que se propone la vinculación del ambiente a áreas como las ciencias sociales y las humanidades. Bajo este referente, encontramos que la educación ambiental en el Colegio La Concepción se encontraba enmarcada en la tercera etapa planteada por el autor, teniendo en cuenta que su trabajo se había limitado a utilizar el reconocimiento de espacios naturales y sus relaciones biológicas físicas y químicas, relacionando su objeto con las ciencias naturales, en la que adicional se establecieron ejes curriculares que incluían el ambiental y modificaban su organización original.

De esta forma se partió de la necesidad de propiciar estrategias diferentes a las tradicionales para abordar la educación ambiental, por lo que se estableció como aspecto relevante la producción de la miniserie llamada "El Capitán Ambiente" la cual ha permitido salir del trabajo interno en la institución, generando acciones mucho más alternativas de gran impacto, haciendo partícipes a mas miembros de la comunidad e incluyendo a otras comunidades. La idea de esta producción audiovisual se fundamentó al identificar que culturalmente se han tenido como referentes diferentes superhéroes, que se presentan como un ejemplo para las sociedades, cuyo perfil está enfocado en ayudar a las comunidades y solucionar las problemáticas convirtiéndose en modelos a seguir. Así, resulto novedoso la construcción de un superhéroe para el ámbito ambiental que no solo se representa en la figura masculina sino que también se visualiza en la femenina.

Esta propuesta no se limitó en permitir la participación a los estudiantes líderes ambientales, sino que permitió la intervención activa de muchos estudiantes y profesores de la institución quienes fueron actores en las diferentes escenas. Como resultado se concibió una producción audiovisual propia del colegio La Concepción que ha traído un mensaje de cuidado y protección de nuestro entorno, identificándose como una excelente 
Bio - grafia. Escritos sobre la Biología y su Enseñanza. ISSN 2027

Edición Extraordinaria. p.p. 503 - 512

Memorias del VIII Encuentro Nacional de Experiencias en Enseñanza de la Biología y la Educación Ambiental. III Congreso Nacional de Investigación en Enseñanza de la Biología.

estrategia de incidencia en la comunidad educativa, por que los estudiantes conocen y se sienten identificados con el súper héroe ambiental y su razón de ser, identificando que el capitán ambiente no los invita a seguirlo si no busca despertar el súper héroe que cada uno lleva dentro, lo que ha aumentado la participación en el grupo, el sentido de pertenencia en relación a su entorno y por ende la multiplicación del mensaje en la comunidad.

Adicional, esta herramienta alternativa de educación ambiental, género en la comunidad educativa un gran impacto reconociendo que estudiantes, profesores y padres de familia reconocían las nuevas formas de educación ambiental en su escuela; motivándose a continuar con el trabajo de recuperación y apropiación del entorno, identificando que ahora sus problemáticas ambientales eran más visibles.

A partir, de este instrumento audiovisual y reconociendo su gran impacto como parte de la estrategia de educación ambiental, se abordó como segunda herramienta de impacto, el diseño de una cartilla ambiental para colorear, identificando que la mayoría de los súper héroes presentan versiones en historieta, por lo que se pensó en trasladar la estrategia audiovisual a la visual, incentivando la imaginación de los estudiantes más pequeños. Así, la cartilla se dirigió a los niños pertenecientes a la educación básica primaria de la comunidad educativa La Concepción buscando desde sus motivaciones despertar en los estudiantes de cortas edades el interés por el cuidado de los recursos y la identificación de las problemáticas ambientales sociales. El contenido de la cartilla ambiental describió la llegada de un súper héroe ambiental a la tierra que al ver la contaminación existente y el maltrato por los seres vivos decide vincularse con estudiantes de un colegio distrital, promoviendo acciones de cuidado de recursos y el cambio de actitudes, logrando que quienes hagan esto se transforme en un súper héroe ambiental. Se resaltaron problemáticas ambientales mundiales que los estudiantes asumían desde su propio contexto y con las cuales se sentían identificados, se evidencio que la contaminación y el desperdicio del agua es una de las problemáticas que más relevancia presenta en la comunidad educativa probablemente por la cercanía que se tiene a la cuenca del rio Tunjuelito. Los estudiantes encontraban en la cartilla la posibilidad de identificarse con un súper héroe ambiental y de esta manera contribuir con propuestas y acciones para el cuidado de los recursos que se encuentran en su entorno inmediato. 
Bio - grafia. Escritos sobre la Biología y su Enseñanza. ISSN 2027

Edición Extraordinaria. p.p. 503 - 512

Memorias del VIII Encuentro Nacional de Experiencias en Enseñanza de la Biología y la Educación Ambiental. III Congreso Nacional de Investigación en Enseñanza de la Biología.

Dentro de todo el desarrollo de esta estrategia ha existido siempre un proceso de auto evaluación de cada una de las acciones realizados lo cual nos ha permitido evidenciar y ser conscientes de aquello que se ha realizado de manera adecuada y de lo que se debe replantear. Así, hemos podido evidenciar que el mensaje sobre la protección y cuidado ambiental que inicialmente se propuso no estaba llegando por completo a los estudiantes debido a que las propuestas se presentaban de forma muy tradicional y repetitivas, por lo que surgió la idea de llegar a ellos de una forma más interesante y motivadora para los miembros de la comunidad.

Finalmente identificamos que esta estrategia de educación ambiental alternativa a la tradicional ha generado un gran impacto en la comunidad educativa, propiciando motivación de los estudiantes para recuperar su entorno; concientización de las familias frente a las problemáticas ambientales de su entorno; el deseo de nuevos estudiantes para pertenecer al proyecto ambiental y el reconocimiento por parte de entidades externas al trabajo realizado ya que esta experiencia se ha socializado en espacios nacionales e internacionales consiguiendo su reconocimiento y generación de trabajos interinstitucionales.

\section{CONCLUSIONES}

En la generación de esta estrategia de educación ambiental en la escuela los medios visuales y audiovisuales, han sido herramientas importantes permitiendo que en su proceso de construcción los estudiantes evalúen las problemáticas ambientales en las que están inmersos, las identifiquen y busquen la forma de cambiarlas.

La revisión de aspectos en los que se posicionaba la educación ambiental en este tipo de comunidad educativa nos permitió concluir que la formulación del mensaje que se quiere enseñar en términos de educación ambiental no se encuentra mal fundamentado desde las políticas y los lineamientos, si no que es la forma de abordarlo la que resulta siendo poco apropiada. Por lo que es necesario variar las formas tradicionales de educación ambiental en la escuela, estableciéndolas de forma independientes al área de conocimiento o formación de los maestros que cotidianamente asumen este reto. Teniendo claro, que no podemos limitarnos a trabajar en posibles soluciones de problemáticas ambientales que nos aquejan en la comunidad desde la misma óptica, por lo que la educación ambiental no se puede seguir limitando a las ciencias naturales. 
Bio - grafia. Escritos sobre la Biología y su Enseñanza. ISSN 2027

Edición Extraordinaria. p.p. 503 - 512

Memorias del VIII Encuentro Nacional de Experiencias en Enseñanza de la Biología y la Educación Ambiental. III Congreso Nacional de Investigación en Enseñanza de la Biología.

Es necesario ampliar este tipo de propuestas al trabajo en educación ambiental con padres de familia y sectores productivos, a fin de contribuir con la minimización del impacto ambiental que se produce en las casas y las empresas. Además, vincular otras instituciones educativas, en las que se visibilice no solo la propuesta si no las problemáticas ambientales.

\section{BIBLIOGRAFIA}

Alcaldía Mayor de Bogotá. (2012). Plan de desarrollo Territorial. 2012-2016, Bogotá humana.

Barbero, J. (2002). La educación desde la comunicación. EDUTEKA - Tecnologías de Información y Comunicaciones para Enseñanza Básica y Media. Editorial Norma.

Duarte J \& Vallejo C. (2013). Propuesta formativa en educación ambiental a partir de las representaciones sociales de ambiente y educación ambiental en el C.E. D. Concepción.

Duarte J., Mojica M. y Calvo, Z. (2013). El ambiente desde lo alternativo. Documento presentado al premio de investigación e Innovación. IDEP.

Gadotti, M. (2000). Pedagogía de la tierra y cultura de la sustentabilidad. Texto retoma ideas tratadas en el libro Pedagogía de la Tierra publicado por la Editora Petrópolis de São Paulo.

González, E. (2000). La transversalidad de la Educación Ambiental en el curriculum de la enseñanza básica. Reflexiones sobre la educación ambiental II. Carpeta Informativa CENEAM. Edita, organismo autónomo Parques Naturales y Ministerio de Medio Ambiente. 
Bio - grafia. Escritos sobre la Biología y su Enseñanza. ISSN 2027

Edición Extraordinaria. p.p. 503 - 512

Memorias del VIII Encuentro Nacional de Experiencias en Enseñanza de la Biología y la Educación Ambiental. III Congreso Nacional de Investigación en Enseñanza de la Biología.

Secretaria de Educación del Distrito - SED. (2012). Proyecto de Educación para la Ciudadanía y la Convivencia (PECC).

Proyecto Educativo Institucional (2009). La Concepción hacia el futuro. Construyendo hechos de vida. CED. La Concepción. Bogotá 